\title{
Introducing qualitative and social science factors in archaeological modelling: necessity and relevance
}

\author{
By M. Vander Linden, M. Saqalli
}

\section{Abstract}

Despite having been a pedigree stretching for several decades, computational approaches remain highly debated in archaeology, hailed by some as the future of the discipline, and discarded by some as a poor, arrogant and overgeneralizing attempt at mimicking the past. This introductory chapter argues that traditional criticisms made at computational models stem from several fundamental misunderstandings. In particular, several archaeologists favouring either a more "social theory" perspective or a more "fieldwork first and avoid generalizing" approach have negatively commented upon what they perceived as simple models when compared to the complex, holistic nature of social life. We argue here that modelling scientific teams, combining archaeologists and modellers, are aware of these complexity and uncertainty and rather prefer to tackle it by explicitly focusing upon a minimal set of epistemological procedures, concepts and parameters, set in an explicitly formal environment. The implications of this epistemological standpoint are evaluated in view of the various contributions to this volume, presented at the end of this contribution.

\section{Key-words}

Archaeology; Computational modelling; agent-based modelling; epistemology; past rural society

\section{Introduction}

The early use of computational modelling and simulations in archaeology can be traced back to the 1960s and 1970s. These can be linked to both new computerizing technologies and concepts, but also to expanding processual paradigms which were dramatically instrumental in reshaping the archaeology discipline as we largely know and practice it still nowadays (Doran and Hodson, 1975).

Whilst the acknowledgement of the role of computational modelling has experienced major fluctuations throughout the 1980s and 1990s (Aldenferer, 1991), parallel to major dissensions within archaeological theory, the use and relevance of computational models in archaeology has exponentially grown over the past couple of decades (for an extensive historical review, see Lake 2014 and for new explorations, see Barceló and del Castillo 2016). The reasons for this resurgence are manifold, and the growing availability of increasingly cheaper and powerful computing hardware must not be underestimated in this process (Lake, 2014; Grosman, 2016).

From a theoretical and methodological point of view, modelling approaches allow to articulate in a formal way numerous and various factors (Shennan and Steele, 2005; Kohler and Gumerman, 2000; Kohler and van der Leeuw, 2007) and, especially in the case of agent-based models, to explore their complex interactions in a non-deterministic way (Cegielski and Roberts, 2016). In this perspective, It is noteworthy that computational models do not differ from so-called literary models, widely used by "traditional" archaeologists, in the sense of "verbal descriptions of a proposed set of causal relationships" (Steele and Shennan 2009: 108).

So, in regard of this apparent proximity, why does computational modelling remain a relatively niche and specialized activity within archaeology? For instance, Lake was able to count only 70 simulation studies having been published between 2001 and 2014, not counting purely conceptual and duplicates of the same model (Lake 2014). One may use as a counter-example the progressive intrication between GIS and geography studies to form one sole discipline, inducing the subsequent equivalent dynamic for geographically related disciplines such as geology, geomorphology or pedology. The same impregnation occurs between biology and related disciplines in one hand and computerized statistics on the other hand.

Of course, because modelling is also a question of practice for apprehending not-so-obvious concepts and acquiring related skills, many archaeologists face difficulties in terms of computer use and coding. Whilst this 
is arguably often the case, successful alternative strategies exist for formalizing the related interdisciplinarity (Etienne et al., 2011), and in numerous instances, computational modelling in archaeology is the outcome of productive, if complex, interdisciplinary projects. We rather hypothesize that more fundamental issues and misunderstandings about the modelling process, deeply rooted in theoretical and epistemological assumptions explain the reluctant engagement of many archaeologists towards computational models.

As any in-depth assessment of either the full breadth of archaeological theory or archaeological computational modelling lies beyond the remits of the present text, the following introduction focuses on what seem to us to be two related issues, namely perception of simplicity vs. simplicity as requirement, and question of adequation of data and simulations.

A further remark is needed. As part of this volume and introduction, our attention is mostly devoted a particular time of computational models, namely agent-based, or multi-agent models (Cegielski and Rogers 2016). It must be stressed that this particular category of models is not, by a long margin, the only one used in archaeology as, for instance, reaction-diffusion models have been very popular when discussing the spread of populations and/or next technologies (Steele 2009 for a recent synthesis). The issues discussed here actually apply to all forms of modelling, though they are perhaps more acute when considered from the point of view of agent-based models.

\section{Model simplicity and data}

For many archaeologists, especially those less familiar with the approach, it seems that the simplicity of models is the most detrimental element, leading to a perception that models are mechanistic, inherently flawed with self-fulfilling prophecies and incapable to encompass in anyway the complexity of both the past and data.

Pointillism vs. impressionism: There is here a simple but meaningful difference about the definition of the seek of truth: as a caricature, one may describe the field archaeologist as a pointillist approach, with a series of field dots, each one providing limited but significant information, eventually leading to a meaningful interpretation through accumulation of a sufficient number of dots. On the other hand, modellers would have an impressionist approach, where an initially gross, blurred but global image would improve along a more detailed process of local dynamics affecting the whole system. Therefore, one side sees models as always false and blurred, while the other side sees dots as - nearly - pointless.

Mimicking and not reproducing: While several archaeologists consider simplification as a threat to the acknowledgement of the inherent complexity of human life, on the contrary modellers see it as a necessary step to get an insight into the very same complexity. In both cases thus, complexity is never challenged, although the ways to assess it profoundly differ. Computational models are, first and foremost, formal thought experiments: their mathematical architecture requires explicit formalism to translate hypotheses and suggested causalities into rules and code, but otherwise cannot, as already stated, have any claim to being intellectually better than literary models. In this sense, and if only for practical reasons, simplification is a necessary step. At least in modellers' mind, models do not aim at reproducing reality, but rather, at best, at encapsulating some properties of reality.

"Kiss" vs. "Kids": The extent and nature of these properties are actually debated within the modelling community itself: some adopt a highly abstract stance and tend to consider models as pure theoretical, thought experiments, an approach often leading to simple models aiming at exploring one given hypothesis and/or process. On the contrary, other modellers consider that models must incorporate a higher level of complexity, leading to the addition of numerous parameters and rules. This somewhat binary dichotomy is often referred to as an opposition between so-called KISS (Keep It Simple Stupid) vs. KIDS (Keep It Descriptive Stupid) models (Edmonds and Moss, 2005; Cegielski and Rogers 2016), and contributions to this volume stand on different sections of this spectrum, including the present authors (compare for instance Saqalli et al. 2016 with Drost and Vander Linden 2018). 
Post hoc ergo propter hoc: This debate on the respective merits of simple vs. complex models is not simply to show that modellers are aware of how complex their object of study is. One may suggest repositioning this debate within the epistemological dialogue regarding the respective positioning of causalities and correlations into a scientific demarche: causalities link parameters through a dynamic, initially qualitatively defined process. But because causalities cannot be proven, they are hypothesised. Testing these hypotheses implies refutation using correlation significance and, therefore, the use of quantitative data.

Quantitative data are an imperative step for replicable science: they define the weight and values of the different parameters which, if non-characterized, would produce fuzzy and useless results. However, defining which parameters are required for archaeology turns out to be an unexpectedly extremely difficult task. For instance, several simulations presented here are spatially-explicit, and therefore include extensive discussions upon the selection and identity of spatial parameters under consideration, including presence/absence of specific features such as rivers, ecological niches or land use categories. As many authors here explain, such debates do occur between proponents of various disciplines, and are, intrinsically, qualitative. Yet, it remains obvious that any simulation space, how complex it is, will never qualify as a proper ecology, or as a landscape lived in and experienced by humans. This discrepancy may appear as a shortcoming of modelling, but is not detrimental since the model never aims at being real.

The second source of misunderstanding between modelers and archaeologists lies in their respective attitude towards data. Indeed, from a modelling perspective, the development of models does not merely rise, as sometimes may be thought by non-practioners, from the sole wish to offer a formal description of past, but as a response to old archaeological problem of the generally low resolution and patchy character of the archaeological record. As stressed in several chapters (Saqalli et al., this volume, O'Brien and Bentley this volume; Carrer et al. this volume), archaeological data are by definition flawed and, following David Clarke's well-known definition, archaeology is the "discipline with the theory and practice for the recovery of unobservable hominid behavior patterns from indirect traces in bad samples" (Clarke 1973: 17). Whilst archaeologists have devised countless tools to account for some of these biases (e.g. taphonomy), eventually it remains impossible to assess how much exactly was lost from the past. The modellers' response to this conundrum is to perform simulations, meaning the creation of "fake" data. The point of the procedure is not to obtain a simulacrum of the past or, worse, the pretense of an alternative past, but rather a continuous environment generated under known conditions. This decision in turn raises further problems as to how to compare such unbiased distribution with patchy archaeological data (see below).

\section{Socio-Anthropology as an unavoidable science}

The development of socio-ecological models, for both past and present situations, relies upon the selection of relevant variables. If the translation from quantitative data to components' behaviour rules is relatively easy, the translation from qualitative traits is yet to be settled. The latter is generally achieved by calculating mean or median values, and variances from various existing datasets, which are then used to set parameters and/or rules in the models. The criteria applied in choosing relevant variables are often less explicit. This is well exemplified in models where environmental factors are often given primacy. Yet numerous studies indicate that anthropological factors (e.g. family organization, inheritance rules, distribution of power amongst lineages or families) can drastically impact upon the environment, sometimes in a counterintuitive way (see for instance Rouchier and Requier-Desjardins, 2000; Polhill et al., 2010; Saqalli et al., 2010). Therefore, we raise the more general question of the inclusion and consideration of qualitative variables into computational models, and, beyond, of the scientific validation of such models. This proposed volume will explore these questions, including the corresponding theoretical and epistemological challenges.

Thanks to inference from better-known situations coming for example from the present time, where one may directly get access to people's beliefs, intentions and rationalities through a mix of socio-anthropological field methodologies (interviews, questionnaires, participatory observations, etc.), we are, mostly, aware of the importance of related factors not only in terms of conceptual weight but more prosaically in terms of evolution of the model. For instance, a society with a one-heir inheritance system in a context of land scarcity or with gender differentiation induces strong and self-amplifying social stratification without further dynamics. But how strong is this influence? Could these factors in general be not crucial enough for NOT being considered 
in socio-ecological models? Thanks to a wide array of paleo-environmental datasets (e.g. dendrochronology, carpology, palynology, anthracology and other data collecting methods on climate and vegetation), access to these elements are far easier. Suggesting causalities between climatic and/or environmental events and archaeological dynamics thus becomes very tempting, but does it pass the test of refutation, such as equivalent environmental events without visible impacts on archaeological records? We assume that it cannot be. As a simple illustration, one may think of important historical events in well-archived societies with no possible connection to environmental dynamics. More globally, let us consider for instance rural societies where demographic density is low and where access to any asset (food, water, shelter, etc.) depends mainly on manpower. Access to manpower in a family is defined by marriages, demography, family organization, extra-family organization and rules, differential access to production activities, etc. In short, one's living and even surviving conditions depend on social rules within the related culture. Finally, one may find difficult to not acknowledge the importance of social networks (e.g. Roux this volume; see also Lemieux, 1976; Collier et al., 2009; Amblard et al., 2010; Filho et al., 2011; Neumann et al., 2011; Gabbriellini, 2014; Neumann and Lotzmann, 2016; Thiriot, 2018).

Once we assume such a position, how can we formalize such non-environmental factors relying on socioanthropological and political factors? How can we assess their integration epistemologically, scientifically and practically? The following chapters offer numerous are proposals for building such an integrative corpus of paradigms and methods.

\section{A brief overview of individual chapters}

The seven chapters in this volume all explore in different ways these many questions. Although a certain emphasis is discernible upon modelling of ecological-economic systems (Saqalli et al. this volume; Barceló et al. this volume; Le Néchet et al. this volume; Carrer et al. this volume), the following chapters explore a wider range including niche construction theory (Bentley and O'Brien this volume), kinship (Read this volume) and cultural transmission (Roux this volume). A majority of chapters includes several authors from varied background, reflecting the aforementioned strong interdisciplinary component of archaeological modelling, though it must be noted that single- and dual-authored chapters all have stem from longer collaborative research projects (e.g. Roux et al. 2018).

The opening chapter by Saqalli and colleagues recalls several points made earlier in this introduction, with further emphasis regarding the status of modelling within archaeology. For instance, they ask whether computational modelling should be considered as an integral part of the discipline, or rather as a highly specialised activity "sub-contracted" to external practitioners. Beyond the obvious relations of power both alternatives imply, they stress the centrality of interdisciplinarity in modelling, not simply because it relies upon different skillsets distributed among various fields, but fundamentally because the research questions being addressed, especially so in rural socio-ecological systems, involve several disciplines and categories of evidence. Such interdisciplinary dialogue relies upon trust and humility between partners (see also Vander Linden 2017), as well as sacrifice on each partner's part. As Saqalli and colleagues put it, the need for sacrificing a portion of the complexity of each object of inquiry is imperative to assure interdisciplinary dialogue, and to achieve the ultimate simplification requested by the very act of modelling. Although simplification is thus a necessary step, the approach adopted by Saqalli and colleagues to account for early European Neolithic farming systems (see also Saqalli et al. 2017) clearly falls towards the KIDS end of the spectrum, complexity being considered as necessary to capture the variability and adaptability of rural socioecological systems. Their chapter provides a series of practical recommendations for the elaboration of such models, including the choice of appropriate analogues for identifying and setting up parameters, and the implementation of the rules governing the behaviour of the model. One of their key points is the dynamic nature of modelling. Indeed, because of their architecture, computational models allow for continuous modification, addition or suppression of parameters. Yet, this complexity comes at a prize, as the multiplicity of parameters renders difficult the evaluation of their confounding effects upon the behaviour of the simulations. Possible solutions include hierarchisation of the parameters, required to avoid the pitfalls of determinism, the combination of factors belonging to different levels, and sensitivity analysis. Alternative pathways include more parsimonious approaches based upon incremental additions of parameters, a process admittedly easier to undertake with KISS models (e.g. Drost and Vander Linden 2018). 
Although focusing on an altogether radically different topic - ethnogenesis - Barceló and colleagues' contribution follows closely similar epistemological and methodological lines to the ones exposed by Saqalli and co-workers. Not only do they illustrate that modelling can address deeply humanistic questions such as identity, and thus need not being limited to economic and environmental issues (see also Kovačević et al. 2015), their chapter also discusses issues related to the way archaeologists routinely describe and transform their data and how modelling can contribute to such continuous methodological renewal. Their extensive and complex review of the literature on ethnicity and identity elegantly illustrates that the apparent simplicity of their model does not stem from unawareness of the issues at stake, but rather of a conscious, deliberate methodological and epistemological reflexion. In this sense, their simulation approach expresses the close links between ethnicity, knowledge transmission and thus historicity. Arguably, reducing ethnicity to a single measure of similarity may seem reductionist, but it provides an effective way to conceptualise otherwise implicit interpretative themes, as well as a quantifiable measure inferred from numerous archaeological data, following David Clarke's famous polythetic definition of archaeological assemblages (Clarke 1968). This explicit reference to Clarke's work provides one of the many testimonies to his long-lasting influence upon the modelling community (e.g. recently Lycett and Shennan 2018). Barceló and colleagues' model also exemplifies how much ethnicity can only be studied in an effective way as the combination of observable practices, seen as a vast array of factors, all articulated in their model design (e.g. food acquisition and exchange, human mobility, varying carrying capacity of the environment). Whilst Barceló and colleagues are dedicated to analyse both simulation results and archaeological data using the same techniques (i.e. similarity indices), this methodological decision leads them to face the aforementioned problem of having to deal with very dissimilar datasets, with models full of data of the one hand, and archaeological data "full of holes" on the other hand, notwithstanding the fact that several variables remain simply out of reach of the resolution provided by archaeological data. This is a recurring crucial issue, and one which both provides the justification of computational models, but which can also be detrimental to their widespread application (beyond being "mere" thought-experiments). There is probably no single answer on how to resolve this tension between "ideal" simulation data and "biased" archaeological data, but the recent literature offers several avenues worth exploring, including sampling of the simulation results mimicking archaeological deposits (Kovačević et al. 2015), Approximate Bayesian Computation (Edinborough et al. 2015), whilst Barceló and colleagues consider the role of bootstrapping.

Bentley and O'Brien also address the fundamental issue of the adequation between archaeological data and the requirements of modelling and statistical approaches. Following upon previous work (O'Brien and Bentley 2015; see also Bentley et al. 2015), their contribution lies firmly in the tradition of evolutionary archaeology and focuses upon niche construction during the European Neolithic, especially the possible coevolution of dairying practices and lactase persistence (see also Itan et al. 2009, Mathieson et al. 2015). The concept of niche construction provides a robust framework for the coherent analysis of concepts otherwise often studied separately, for instance cultural transmission and food production systems, thus re-affirming the potential for computational modelling to offer efficient tools to discuss unexpected series of relationships and to bridge the compartmentalisation of disciplines. Rather than adopting a simulation approach strictly speaking, Bentley and O'Brien explore the relevance of Granger causality, a linear modelling technique used to assess the incremental predictive value of time series. The advantage of such technique lies in the limited number of variables being considered (here only two), but the drawback is the extreme demands in terms of data required to fix the equations. Despite being arguably one of the richest archaeological records available across the globe, with extensive datasets covering radiocarbon dates, cemeteries and mortality profiles, and settlement patterns, Bentley and O'Brien end up concluding that available data for European Neolithic periods do not match up the requirements of the matrix.

Le Néchet and colleagues' chapter also weaves together modelling and food-production systems, in this case through the lens of the Bantu expansion, and in particular the interaction between Bantu farmers and forest foragers. A combination of archaeological, linguistic and genetic data provides them with competing hypotheses regarding the direction and specifics of the migration of Bantu-speaking farming populations, and especially the role of non-Bantu foraging communities in mediating this process. Informed by historical and ethnographic resources, they stress the symbiotic relationship between both communities and lifeways, focused upon two distinct co-existing ecological niches. In this respect, the African situation described here markedly differs from more European-centric case-studies and simulations, which often consider foragers 
and farmers as either antagonistic entities, or as stages in a linear evolutionary process ultimately leading to the dominance of the latter over the former (e.g. Aoki et al. 1996). Despite this fundamental difference, their model also lies in the continuity of a long tradition of computational models exploring demographic expansion, often based upon reaction-diffusion equations and occurring in an empty, unrealistic landscape (see Steele 2009, Vander Linden and Silva 2018; noticeable exceptions include Davison et al. 2005, Fort et al. 2012). In contrast to such equations, Le Néchet and colleagues rather use a multi-agent system, not simply for the complexity inherent to such computational tools, but, because these are designed to explore emergent properties and thus lack any central control mechanism, echoing expectations regarding the historical situation of the Bantu spread. Interestingly, Le Néchet and colleagues explicitly wish to position their H.U.M.E. model (HUman Migration and Environment) in the middle of the KISS / KIDS dichotomy through the incorporation of both generic and specific traits. As a result, their model incorporates parameters related to food resources and productivity, group fission, technological innovation, and group interaction (including competition and imitation). Rather than never-endingly debating upon what constitutes the minimum requirements for a model to be complex or realistic enough, there is, as Le Néchet and colleagues explain, more to be gained by using computational model as the basis for dynamic interdisciplinary dialogue (see also Saqalli et al. this volume; Carrer et al. this volume). Le Néchet and colleagues for instance depict how earlier versions of their model, and the underlying decisions structuring them, are questioned and transformed through interactions between various participants, eventually leading to the inclusion of several new variables (e.g. modelling two populations using same parameters but set with two distinct sets of values, spatially-explicit environment considering distinct ecologies). Overall, they offer the description of an entire methodological protocol, designed to incorporate differences traditions of conceptualising and analysing data.

Read's chapter may, at first sight, seem to stand out in this volume, as it does provide neither computational model, nor any computer code, nor any simulation. Elsewhere, though, he has provided a detailed demographic simulation of hunter-gatherer societies (Read 1998; see also Read and LeBlanc 2003). In his simulation, he shows that hunter-gatherer societies will have a stabilized population size substantially below carrying capacity (with carrying capacity based on the limitations of the resource procurement systems of hunter-gatherer groups) in regions with a low density of resources and a stabilized population size close to carrying capacity for groups in regions with a high density of resources. This leads to the prediction that intergroup violence is more likely for hunter-gatherer groups in regions with a high resource density. This prediction has been substantiated for hunter-gatherer groups in Australia (Read 2009). In this simulation, Read also showed that if marriages among the !Kung San are consistent with their cultural kinship rules regarding proper and improper marriages, then there would be de facto residence group marriage exogamy even though there is no cultural rule requiring that marriages be exogamous with respect to one's residence group. This shows the value of simulations for working out the behavioural consequences of cultural rules, thereby adding to the ethnographic record. With regard to the kinship terminology systems that Read discusses in this chapter, he has also developed (in conjunction with Michael D. Fischer at Kent University, UK; see Read, Fischer and Leaf 2013) an extensive computer model, called Kinship Algebraic Expert System (KAES), for implementing the algebraic analysis of kinship terminology systems that he discusses in his chapter (Read 2006). The computer model makes it possible for the algebraic analysis of a kinship terminology to be carried out even without a background in the formalism of abstract algebras. In this chapter, his contribution demonstrates how mathematically-driven simplification, implemented in the KAES computer program, offers in-depth insights into such a seemingly complex and fundamental human process as kinship. Read shows how the specificity of human kinship systems rests upon their computational properties by introducing a fundamental qualitative distinction between interaction systems based upon individuals on the one hand, and relation-based social systems on the other hand. Read's argument rests upon the characterisation and description of the evolutionary sequence from genealogical connections (e.g. mother and father relationships) to the symbolic system of kinship relations.

Roux's chapter explores divergences between existing archaeological approaches on cultural transmission and technological change, in particular the respective assumptions of computational models rooted in evolutionary thinking, analytical sociology and the long tradition of ethnoarchaeological research on technology, especially the French chaîne opératoire approach. Roux's criticisms of evolutionary model lie upon the measure of evolution processes independently of the social context into which they take place, as 
well as upon morphometric traits which, despite having the advantage of being easy to quantify, are known to be poor markers of transmission and prone to rapid, quasi-stochastic changes. By contrast, Roux's preference lies with technical traits, as extensive ethnoarchaeological research demonstrates that their acquisition by social agents is tightly associated with social learning. For instance, pottery forming techniques are often acquired over a long period of time, required to master the necessary motor skills (Roux and Corbetta, 1989; Roux et al. 1995 for a non-pottery example), and are thus intrinsically more stable than other parts of the chaîne opératoire. In this sense, the characterisation of the transmission of any technology becomes directly linked to the evaluation of the social structure within which learning occurs. Such consideration for both the content of what is being transmitted and the associated mechanisms of social interaction finds strong parallels with analytical sociology, a field with a long tradition of involvement with simulation approaches, especially network models. The approach suggested here however qualitatively differs from most archaeological applications of network analysis, based on the assumption that similarity, interaction and exchange follow a linear relationship. Given that such material similarities can be the outcome of distinct processes, Roux insists upon the need to select as appropriate and as robust as possible variables or proxies for the subject to be modelled and explained. In this perspective, the selection of relevant proxies is geared at highlighting the relational structure of societies and then using reference sociological models providing explanation of evolution processes to analog relational structures of societies. Here, Roux's epistemological and methodological reflection is put to practice by using the Late Chalcolithic period (4500-3900 cal BC) in the Levant as case-study, eventually demonstrating the leading role of longlasting social network structures in shaping processes of technological innovation and transfer. Although the modelling component is less apparent in her chapter, more explicit considerations can be found in recent publications by her research group (e.g. Manzo et al. 2018).

The last contribution to this volume, by Carrer and collaborators, combines computational modelling, considered as an exploratory framework, ethnoarchaeology, seen as a robust source of analogues for setting up parameters of the models, and historical data on land use in the North Italian Alps, used to validate the results of the simulation. Their starting point lies in the driving role of both environmental and human factors in shaping land use patterns, in particular in mountainous landscapes. Contrasting the long history of human presence in mountains, only accessible through low resolution archaeological and paleo-ecological data, with the comparatively short-term historical ecological knowledge, they advocate the use of computational modelling to further our knowledge of land use over the longue durée. As many other chapters, their methodology is intrinsically interdisciplinary, with a greater emphasis upon the essential role of ethnoarchaeologically documented analogues to set up parameters and calibrate simulations (see also Lancelotti et al. 2017). Here they offer two different models, reflecting two contrasted land use strategies (i.e. local subsistence relying upon cereal cultivation and cattle husbandry, and intensive dairy-focused cattle husbandry). Both models are spatially-explicit and incorporate different ecological niches, and corresponding human practices and carrying capacity, much in line with similar decisions found elsewhere in this volume (especially Saqalli et al. this volume, Barceló et al. this volume, Le Néchet et al. this volume). Although admittedly - and explicitly simple - both models perform rather well, providing reasonable fits with independent historical land use data.

\section{Conclusion}

Although the contributions assembled here cover a range of theoretical approaches and topics, they all share core concerns related to the tension arising between the need for simple models and the inclusion of complex, qualitatively demanding parameters, or between "ideal" simulation and "patchy" archaeological data. Another recurrent theme, inherently linked to the first issue, lies in the development of spatially-explicit models, especially when dealing with rural ecological systems. As previously said, there is no one-size-fits-all solution to be sought or found in these pages, but, more prosaically, numerous methodological and practical solutions. It would however be unrealistic to put such stress upon the modelling community, including its producers and consumers, to come with such magical recipes: the same way archaeological sites require constant development of new, and adaptation of long-established digging techniques, computational modelling remains a work in progress. 
In this sense, we cannot insist more how much computational modelling provides an exciting venue for future archaeological research, especially given its formal requirements and its near-intrinsic interdisciplinary. Although we therefore extend Lake's enthusiasm and call for further development and popularisation of modelling in archaeology (Lake, 2014), at the same time we are not advocating for modelling to become a norm or standard. Firstly, the approach is not appropriate for all dimensions of archaeological practice. Secondly, and despite the fact that computational modelling is not locked in any brand of archaeology, calling for the normalisation of modelling would not be in tune with the diversity of theoretical approaches which has always be the hallmark of the discipline.

Yet we are confident that the variety of topics and approaches exposed here will provide another supplementary step in clearing some of the existing misunderstandings between archaeologists and modelling community, and help foster new work. As argued earlier, the conditions for the successful integration of qualitative factors in modelling extend well beyond the immediate needs of modellers, but have profound implications for archaeological reasoning, including our ability to excavate, analyse and share data.

\section{References}

Amblard, F., Geller, A., Neumann, M., Srbljinovic, A., Wijermans, N., 2010. Analyzing Social Conflict via Computational Social Simulation: A Review of Approaches. NATO Science for Peace \& Security Series 75, 141. https://doi.org/10.1111/j.1468-2486.2009.00910.x

Aoki K., Shida M., Shigesada N. 1996. Travelling Wave Solutions for the Spread of Farmers into a Region Occupied by Hunter-Gatherers. Theoretical population biology, 50, 1, 1-17.

Barceló J.A., del Castillo F. 2016. Simulating prehistoric and ancient worlds, Springer Computational Social Sciences. Springer, USA.

Barceló J.A., Del Castillo F., Del Olmo R., Mameli L., Miguel Quesada F. J., Poza D., Vila X. 2015. Simulating Patagonian Territoriality in prehistory: Space, Frontiers and networks among hunter-gatherers. In: Wurzer G., Kowarik K. Reschreiter H. (eds.) Agent-based Modeling and Simulation in Archaeology. Springer ed., BerlinNew York. Advances in geographic Information Science. 243-289. DOI 10.1007/978-3-319-00008-4_10.

Bentley R. A., O'Brien M. J., Manning K., Shennan S. 2015. On the relevance of the European Neolithic. Antiquity, 89, 347, 1203-1210.

Cegielski W. H., Rogers J. D. 2016. Rethinking the role of agent-based modeling in archaeology. Journal of Anthropological Archaeology, 41, 283-298.

Clarke D.L. 1968. Analytical archaeology. Methuen, London.

Clarke D.L. 1973. Archaeology: the loss of innocence. Antiquity, 47, 6-18.

Collier, N., Boedhihartono, A.K., Sayer, J., 2009. Indigenous livelihoods and the global environment: Understanding relationships. Presented at the 18th World IMACS / MODSIM Congress, pp. 2833-2839.

Davison K., Dolukhanov P., Sarson G. R., Shukurov A. 2006. The role of waterways in the spread of the Neolithic. Journal of Archaeological Science, 33, 5, 641-652.

Drost C. J., Vander Linden, M. 2018. Toy Story: Homophily, transmission and the use of simple simulation models for assessing variability in the archaeological record. Journal of Archaeological Method and Theory, https://doi.org/10.1007/s10816-018-9394-y

Edinborough K., Crema E. R., Kerig T., Shennan S. 2015. An ABC of lithic arrowheads: a case study from southeastern France. In Brink C., Hydén S., Jennbert K., Larsson L., Olausson D. (eds). Neolithic Diversities Perspectives from a conference in Lund, Sweden. Acta Archaeologica Lundensia 65, 213-223. 
Edmonds B., Moss S., 2005. From KISS to KIDS: an "anti-simplistic" modelling approach. Lecture Notes in Artificial Intelligence 34, 130-144.

Etienne M., DuToit D., Pollard S. 2011. ARDI: a co-construction method for participatory modelling in natural resources management. Ecology \& Society 16, 44.

Filho, H.S.B., Neto, F.B., Fusco, W., 2011. Migration and social networks - An explanatory multievolutionary agent-based model. In: Intelligent Agent (IA), 2011 IEEE Symposium. Intelligent Agent (IA), 2011 IEEE Symposium on, pp. 1-7. https://doi.org/10.1109/IA.2011.5953616

Fort J., Pujol T., Vander Linden M. 2012. Modelling the Neolithic transition in the Near East and Europe. American Antiquity 77; 2, 203-219.

Gabbriellini, S., 2014. The Evolution of Online Forums as Communication Networks: An Agent-Based Model. Revue française de sociologie 55, 805-826. https://doi.org/10.3917/rfs.554.0805

Isern N., Fort J., Vander Linden M. 2012. Space competition and time delays in human range expansions: application to the Neolithic transition. PloS one, 7, 12, e51106. doi:10.1371/journal.pone.0051106

Itan Y., Powell A., Beaumont M. A., Burger J., Thomas M. G. 2009. The origins of lactase persistence in Europe, Plos Computational Biology 5(8),: e1000491 doi:10.1371/journal.pcbi.1000491

Kohler T. A., Gumerman G. J. 2000. Dynamics in human and primate societies: Agent-based modeling of social and spatial processes. Oxford University Press, Oxford, UK.

Kohler T. A., van der Leeuw S.E. 2007. The model-based archaeology of socionatural systems. Oxbow Books Ltd, Oxford, UK.

Kovačević M., Shennan S., Vanhaeren M., d'Errico F., Thomas M. G. 2015. Simulating geographical variation in material culture: were early modern humans in Europe ethnically structured? In: Learning Strategies and Cultural Evolution during the Paleolithic. Springer, Tokyo. 103-120.

Lake M. W. 2014. Trends in archaeological simulation. Journal of Archaeological Method and Theory. 21, 258-287.

Lancelotti C., Negre J., Alcaina Mateos J. Carrer F. 2017. Intra-site spatial analysis in ethnoarchaeology. Environmental Archaeology, 22(4) https://doi.org/10.1080/14614103.2017.1299908.

Lemieux, V., 1976. L'articulation des réseaux sociaux. Recherches sociographiques 17, 247-260. https://doi.org/10.7202/055716ar

Lycett S. J., Shennan S. J. 2018. David Clarke's analytical archaeology at 50. World Archaeology. doi:10.1080/00438243.2018.1470561.

Manzo G., Gabbriellini S., Roux V. Nkirote M'Mbogori F. 2018. Complex contagions and the diffusion of innovations: evidence from a small-N study. Journal of archaeological method and theory. https://doi.org/10.1007/s10816-018-9393-z

Mathieson I., Lazaridis I., Rohland N., Mallick S., Patterson N., Roodenberg S. A., et al. 2015. Genome-wide patterns of selection in 230 ancient Eurasians. Nature, 528, 7583, 499-503.

Neumann, M., Braun, A., Heinke, E.M., Saqalli, M., Srbljinovic, A., 2011. Challenges in Modelling Social Conflicts: Grappling with Polysemy. Journal of Artificial Societies \& Social Simulations 14, 9. 
Neumann, M., Lotzmann, U., 2016. Simulation and Interpretation: A Research Note on Utilizing Qualitative Research in Agent-based Simulation. Int J Swarm Intel Evol Comput 5. https://doi.org/10.4172/2090$\underline{4908.1000129}$

O'Brien M. J., Bentley R. A. 2015. The role of food storage in human niche construction: An example from Neolithic Europe. Environmental Archaeology, 20, 4, 364-378.

Polhill G.J., Sutherland L.A., Gotts N.M., 2010. Using Qualitative Evidence to Enhance an Agent-Based Modelling System for Studying Land Use Change. Journal of Artificial Societies \& Social Simulations 13, 10.

Read D. 1998. Kinship Based Demographic Simulation of Societal Processes. Journal of Artificial Societies and Social Simulation. www.soc.surrey.ac.uk/JASSS/1/1/1.html

Read D., LeBlanc S. 2003. Population Growth, Carrying Capacity, and Conflict. Current Anthropology. 44, 1, 59-85.

Read D. 2006 Kinship Algebra Expert System (KAES): A Software Implementation of a Cultural Theory. Social Science Computer Review. 24, 1, 43-67.

Read D. 2009 Agent-based and multi-agent simulations: coming of age or in search of an identity? Computational and Mathematical Organization Theory. 16, 329-347.

Read D., Fischer M. Leaf M. 2013. What are kinship terminologies, and why do we care? A computational approach to analysing symbolic domains. Social Science Computer Review. 31, 1, 16-44.

Rouchier J., Requier-Desjardins M., 2000. La modélisation comme soutien à l'interdisciplinarité en recherche-développement. Une application au pastoralisme soudano-sahélien. Nature, Sciences \& Société. 8, 61-67.

Roux V., Bril V. Dietrich G. 1995. Skills and learning difficulties involved in stone knapping: the case of stonebead knapping in Khambat, India. World Archaeology 27: 63-87.

Roux V., Corbetta D. 1989. The potter's wheel. Craft specialization and technological competence. Oxford: IBH publishing.

Roux V., Bril B., Karasik, A. 2018. Weak ties and expertise: crossing technological boundaries. Journal of Archaeological Method and Theory. https://doi.org/10.1007/s101816-018-9397-9

Saqalli M., Gérard B., Bielders C.L., Defourny P., 2010. Testing the impact of social forces on the evolution of Sahelian farming systems: A combined agent-based modeling and anthropological approach. Ecological Modelling 221, 2714-2727. https://doi.org/10.1016/j.ecolmodel.2010.08.004

Saqalli M., Salavert A., Bréhard S., Bendrey R., Vigne J.-D., Tresset A. 2014. Revisiting and modelling the woodland farming system of the early Neolithic Linear Pottery Culture (LBK), 5600-4900 BC. Vegetation History and Archaeobotany, 23, S1, 37-50.

Shennan S., Steele J. 2005. The archaeology of human ancestry power, sex and tradition. Routledge, London, London, UK.

Steele J. 2009. Human dispersals: mathematical models and the archaeological record dispersal models and case studies: Fisher-Skellam-KPP. Human biology, 81, 121-140.

Steele J., Shennan S. 2009. Introduction: demography and cultural macroevolution. Human biology, 81, 105119. 
Thiriot S., 2018. Word-of-mouth dynamics with information seeking: Information is not (only) epidemics. Physica A: Statistical Mechanics and its Applications 49, 2, 418-430. https://doi.org/10.1016/i.physa.2017.09.056

Vander Linden M. 2017. Reaction to a reactionary text. Norwegian Archaeological Review, 50, 2, 127-129.

Vander Linden M., Silva, F. 2018. Comparing and modeling the spread of early farming across Europe. PAGES newsletter 26, 1, 28-29. 